\title{
On a maximal subgroup of the orthogonal group $\mathrm{O}_{8}^{+}(3)$
}

\author{
D. M. Musyoka \\ Kenyatta University, Kenya \\ L. N. Njuguna \\ Kenyatta University, Kenya
}

A. L. Prins

Nelson Mandela University, South Africa

and

L. Chikamai

Kibabii University, Kenya

Received: March 2021. Accepted : September 2021

\begin{abstract}
The orthogonal simple group $\mathrm{O}_{8}^{+}(3)$ has three conjugacy classes of maximal subgroups of the form $3^{6}: L_{4}(3)$. These groups are all isomorphic to each other and each group has order 4421589120 with index 1120 in $O_{8}^{+}(3)$. In this paper, we will compute the ordinary character table of one of these classes of maximal subgroups using the technique of Fischer-Clifford matrices. This technique is very efficient to compute the ordinary character table of an extension group $\bar{G}=N . G$ and especially where the normal subgroup $N$ of $\bar{G}$ is an elementary abelian p-group. The said technique reduces the computation of the ordinary character table of $\bar{G}$ to find a handful of so-called Fischer-Clifford matrices of $\bar{G}$ and the ordinary or projective character tables of the inertia factor groups of the action of $\bar{G}$ on $N$.
\end{abstract}

MSC (2019): Primary: 20C15; Secondary: 20C40.

Keywords: Coset analysis, Fischer-Clifford matrices, split extension, inertia factor, character table, fusion map, restriction of characters. 


\section{Introduction}

The orthogonal group $O_{8}^{+}(3)$ of order $4952179814400=2^{12} \cdot 3^{12} \cdot 5^{2} .7 .13$ has 27 conjugacy classes of maximal subgroups [8]. Among the maximal subgroups of $O_{8}^{+}(3)$ are three non-conjugate but isomorphic subgroups $\bar{G}_{1}$, $\bar{G}_{2}$ and $\bar{G}_{3}$ of the form $3^{6}: L_{4}(3)$ with order 4421589120 and index 1120 in $\mathrm{O}_{8}^{+}(3)$. The aim of this paper is to compute the Fischer-Clifford matrices and hence the character table of $\bar{G}_{1}$, the first group of the three as they appear in the ATLAS [8]. For this purpose, the Fischer-Clifford matrices technique which is based on Clifford theory and was developed by Bernd Fischer [9] is used. The group $3^{6}: L_{4}(3)$ which we shall now denote by $\bar{G}$ is a split-extension of $N=3^{6}$, the vector space of dimension 6 over $G F(3)$, by the linear group $G=L_{4}(3) \cong O_{6}^{+}(3)$.

Let $\bar{G}=N: G$ be a split extension of $N$ by $G$, where $N$ is a vector space of dimension $n$ over $G F(p)$, for a prime $p$, on which a linear group $G$ acts naturally. The Fischer-Clifford technique involves the construction of a non-singular matrix $M(g)$ for each conjugacy class representative $g$ of $G$, which together with the fusion maps and ordinary character tables of some subgroups of $G$, called the inertia factor groups, are used to assemble the complete ordinary character table of $\bar{G}$.

The Fischer-Clifford matrix $M(g)$ is partitioned row-wise into blocks, where each block corresponds to an inertia group $\bar{H}_{i}$ of $\theta_{i} \in \operatorname{Irr}(N)$ in $\bar{G}$. Using the columns of the character tables of the inertia factors $H_{i} \cong \frac{\bar{H}_{i}}{N}$ which correspond to classes of $H_{i}$ which fuse to the class $[g]$ of $G$ and multiplying these columns by the rows of the the Fischer-Clifford matrix $M(g)$ that correspond to $H_{i}$, a portion of the character table of $\bar{G}$ which is in the block corresponding to $\bar{H}_{i}$ for the classes that come from the coset $N g$ is constructed. The character table of $\bar{G}$ is thus divided row-wise into blocks, where each block corresponds to an inertia group $\bar{H}_{i}=N: H_{i}$. The reader is referred to [2], [15], [16], [19], [20] and [21] for more literature on this technique. A brief theoretical background of the Fischer-Clifford theory is given in Section 2.

In Section 3, the coset analysis technique [17] is used to determine the conjugacy classes of $\bar{G}$. In Sections 4 and 5 the inertia factor groups $H_{i}$ and their fusion maps into $G=L_{3}(4)$ are computed. The Fischer-Clifford matrices of $\bar{G}=3^{6}: L_{4}(3)$ are determined in Section 6 and the associated 
ordinary character table of $\bar{G}$ is to be found in Section 7 . The technique of set intersection of characters (see [1], [17], [18]) is mainly used to compute the fusion of the conjugacy classes of $\bar{G}=3^{6}: L_{4}(3)$ into $O_{8}^{+}(3)$. Most of our computations are carried out with the computer algebra systems MAGMA [7] and GAP [11] and the notation of ATLAS is mostly followed.

\section{Theory of Fischer-Clifford Matrices}

Let $\bar{G}=N$ : $G$ be a split extension of $N$ by $G$. Then for $\theta \in \operatorname{Irr}(N)$, we define $\bar{H}=\left\{x \in \bar{G} \mid \theta^{x}=\theta\right\}=I_{\bar{G}}(\theta)$ and $H=\left\{x \in G \mid \theta^{x}=\theta\right\}=I_{G}(\theta)$ where $I_{\bar{G}}(\theta)$ is the stabilizer of $\theta$ in the action of $\bar{G}$ on $\operatorname{Irr}(N)$, we have that $I_{\bar{G}}(\theta)$ is a subgroup of $\bar{G}$ and $N$ is normal subgroup in $I_{\bar{G}}(\theta)$. Also $\left[\bar{G}: I_{\bar{G}}(\theta)\right]$ is the size of the orbit containing $\theta$. Then it can be shown that $\bar{H}=N: H$, where $\bar{H}$ is the inertia group of $\theta$ in $\bar{G}$. The inertia factor $\bar{H} / N \cong H$ can be regarded as the inertia group of $\theta$ in the factor group $\bar{G} / N \cong G$. Define $\theta^{g}$ by $\theta^{g}(n)=\theta\left(g n g^{-1}\right)$ for $g \in \bar{G}, n \in N$, then $\theta^{g} \in \operatorname{Irr}(N)$. We say that $\theta$ is extendible to $\bar{H}$ if there exists $\varphi \in \operatorname{Irr}(\bar{H})$ such that $\varphi \downarrow N=\theta$. If $\theta$ is extendible to $\bar{H}$ then by Gallagher [10], we have $\left\{\varphi \mid \varphi \in \operatorname{Irr}(\bar{H}),<\varphi \downarrow_{N}\right.$, $\theta>\neq 0\}=\{\bar{\beta} \varphi \mid \beta \in \operatorname{Irr}(\bar{H} / N)\}$, where $\bar{\beta} \in \operatorname{Irr}(\bar{H})$ is a lifting for $\beta$ into $\bar{H}$. Let $\bar{G}$ have the property that every irreducible character of $N$ can be extended to its inertia group. Now let $\theta_{1}=1_{N}, \theta_{2}, \ldots, \theta_{t}$ be representatives of the orbits of $\bar{G}$ on $\operatorname{Irr}(N), \bar{H}_{i}=I_{\bar{G}}\left(\theta_{i}\right), 1 \leq i \leq t, \varphi_{i} \in \operatorname{Irr}\left(\bar{H}_{i}\right)$ be an extension of $\theta_{i}$ to $\bar{H}_{i}$ and $\bar{\beta} \in \operatorname{Irr}\left(\bar{H}_{i}\right)$ such that $N \subseteq \operatorname{Ker}(\bar{\beta})$. Then it can be shown that

$$
\begin{array}{rlc}
\operatorname{Irr}(\bar{G}) & =\bigcup_{i=1}^{t}\left\{\left(\bar{\beta} \varphi_{i}\right)^{\bar{G}} \mid \bar{\beta} \in \operatorname{Irr}\left(\bar{H}_{i}\right), N \subseteq \operatorname{Ker}(\bar{\beta})\right\} \\
& = & \bigcup_{i=1}^{t}\left\{\left(\bar{\beta} \varphi_{i}\right)^{\bar{G}} \mid \beta \in \operatorname{Irr}\left(\bar{H}_{i} / N\right)\right\}
\end{array}
$$

Hence the irreducible characters of $\bar{G}$ will be divided into blocks, where each block corresponds to an inertia group $\bar{H}_{i}$. Let $H_{i}$ be the inertia factor group and $\varphi_{i}$ be an extension of $\theta_{i}$ to $\bar{H}_{i}$. Take $\theta_{1}=1_{N}$ as the identity character of $N$, then $\bar{H}_{1}=\bar{G}$ and $H_{1} \cong G$. Let $X(g)=\left\{x_{1}, x_{2}, \ldots, x_{c(g)}\right\}$ be a set of representatives of the conjugacy classes of $\bar{G}$ from the coset $N \bar{g}$ whose images under the natural homomorphism $\bar{G} \rightarrow G$ are in $[g]$ and we take $x_{1}=\bar{g}$. We define,

$$
R(g)=\left\{\left(i, y_{k}\right) \mid 1 \leq i \leq t, H_{i} \cap[g] \neq 0,1 \leq k \leq r\right\}
$$

and we note that $y_{k}$ runs over representatives of the conjugacy classes of elements of $H_{i}$ which fuse into $[g]$ in $G$. Then we define the Fischer-Clifford 
matrix $M(g)$ by $M(g)=\left(a_{\left(i, y_{k}\right)}^{j}\right)$, where $a_{\left(i, y_{k}\right)}^{j}=\sum_{l}^{\prime} \frac{\left|C_{\bar{G}}\left(x_{j}\right)\right|}{\left|C_{\overline{H_{i}}}\left(y_{l_{k}}\right)\right|} \varphi_{i}\left(y_{l_{k}}\right)$ with columns indexed by $X(g)$ and rows indexed by $R(g)$ and where $\sum_{l}^{\prime}$ is the summation over all $l$ for which $y_{l_{k}} \sim x_{j}$ in $\bar{G}$. Then the partial character table of $\bar{G}$ on the classes $\left\{x_{1}, x_{2}, \ldots, x_{c(g)}\right\}$ is given by

$$
\left(\begin{array}{c}
C_{1}(g) M_{1}(g) \\
C_{2}(g) M_{2}(g) \\
\vdots \\
C_{t}(g) M_{t}(g)
\end{array}\right)
$$

where the Fischer-Clifford matrix

$$
M(g)=\left(\begin{array}{c}
M_{1}(g) \\
M_{2}(g) \\
\vdots \\
M_{t}(g)
\end{array}\right)
$$

is divided into blocks $M_{i}(g)$ with each block corresponding to an inertia group $\bar{H}_{i}$ and $C_{i}(g)$ is the partial character table of $H_{i}$ consisting of the columns corresponding to the classes that fuse into $[g]$ in $G$. We can also observe that the number of irreducible characters of $\bar{G}$ is the sum of the number of irreducible characters of the inertia factors $H_{i}$ 's. For complete information on the properties of Fischer-Clifford matrices the reader is referred to [2], [15], [16], [19], [20] and [21]. The group $\bar{G}=3^{6}: L_{4}(3)$ is a split extension with $3^{6}$ abelian and therefore by Mackey's theorem (see Theorem 5.1.15 in [18]), we have that each irreducible character of $3^{6}$ can be extended to its inertia group in $\bar{G}$. With this theoretical assertion in mind, the character table of $\bar{G}=3^{6}: L_{4}(3)$ is now going to be determined using the above outline.

\section{The Conjugacy Classes of $\bar{G}=3^{6}: L_{4}(3)$}

In this section, the method of coset analysis is used to determine the conjugacy classes of the elements of $\bar{G}=3^{6}: L_{4}(3)$. This method was developed and first used by Moori in [17] and since then, it has been used by many other researchers to compute the conjugacy classes of groups of extension type. The reader is referred to [3] and [4] for recent application of this technique. By making use of the standard generators from the online ATLAS of Group Representations [25], the groups $P=O_{8}^{+}(3)$ 
and $\bar{G}$ are represented as permutations on 1080 points in MAGMA. The command "IsMaximal $(P, \bar{G})$;" confirms that $\bar{G}$ is a maximal subgroup of $P$. Proceeding with the commands, " $a, b:=$ ChiefSeries $(\bar{G}) ; ", " N:=$ $a[2]$ ", "M: $=$ GModule $(\bar{G}, N) ; "$ and "M:Maximal;" the group $G=L_{4}(3)$ is constructed as matrix group of degree 6 over $G F(3)$. Note that $N$ is the only elementary abelian 3 -group of order 729 in $\bar{G}$. The following two $6 \times 6$ matrices $g_{1}$ and $g_{2}$ of orders 2 and 12, respectively, are obtained as the generators of $G$.

$$
g_{1}=\left(\begin{array}{cccccc}
0 & 1 & 0 & 0 & 0 & 0 \\
1 & 0 & 0 & 0 & 0 & 0 \\
0 & 0 & 0 & 0 & 1 & 0 \\
0 & 0 & 2 & 1 & 1 & 0 \\
0 & 0 & 1 & 0 & 0 & 0 \\
1 & 2 & 1 & 0 & 2 & 1,
\end{array}\right) \quad g_{2}=\left(\begin{array}{llllll}
0 & 0 & 1 & 0 & 0 & 0 \\
0 & 0 & 0 & 1 & 0 & 0 \\
2 & 1 & 1 & 1 & 0 & 0 \\
2 & 0 & 0 & 1 & 1 & 0 \\
0 & 0 & 0 & 0 & 0 & 1 \\
1 & 0 & 1 & 0 & 1 & 0
\end{array}\right)
$$

The 29 conjugacy classes of $G=\left\langle g_{1}, g_{2}\right\rangle$ are computed within GAP.

\subsection{The Action of $G=L_{4}(3)$ on $3^{6}$}

Let $\bar{G}=3^{6}: L_{4}(3)$ be the split extension of $G=\left\langle g_{1}, g_{2}\right\rangle$ by $N=3^{6}$, where $N$ is considered as a vector space $V_{6}(3)$ of dimension 6 over $G F(3)$. Also, $N \cong V_{6}(3)$ as a $G$-module of $G=\left\langle g_{1}, g_{2}\right\rangle$ is irreducible. Using GAP, it turns out that the action of $G=L_{4}(3)$ on $N=3^{6}$ has four orbits of lengths 1,234, 234 and 260 with corresponding point stabilizers $P_{1}, P_{2}, P_{3}$ and $P_{4}$.

\subsection{Permutation Character of $G=L_{4}(3)$ on $3^{6}$}

Checking the indices of maximal subgroups of $G=L_{4}(3)$ in the ATLAS [8], $P_{2}$ and $P_{3}$ sit maximally inside the maximal subgroups with the structure $U_{4}(2): 2$ while $P_{4}$ sits maximal inside the maximal subgroup $3^{4}: 2\left(A_{4} \times A_{4}\right) .2$ of $G$. It follows that $P_{1}=L_{4}(3), P_{2}=U_{4}(2), P_{3}=U_{4}(2)$ and $P_{4}=3^{4}: 2\left(A_{4} \times A_{4}\right)$ of indices $1,234,234$ and 260 respectively in $L_{4}(3)$. We will now determine, with the use of the permutation character $\chi\left(L_{4}(3) \mid 3^{6}\right)$ of $G$ on $N$ whether $P_{2}$ and $P_{3}$ are sitting separately inside one of the two classes of $U_{4}(2): 2$ or both are in one of the classes of $U_{4}(2): 2$. The permutation character $\chi\left(L_{4}(3) \mid 3^{6}\right)=1+I_{P_{2}}^{L_{4}(3)}+I_{P_{3}}^{L_{4}(3)}+I_{P_{4}}^{L_{4}(3)}$ of $G$ acting on $N$ is now going to be computed, where $I_{P_{2}}^{L_{4}(3)}, I_{P_{3}}^{L_{4}(3)}$ and $I_{P_{4}}^{L_{4}(3)}$ are the identity characters of $P_{2}, P_{3}$ and $P_{4}$ induced to $G$ respectively. To determine 
$I_{P_{2}}^{L_{4}(3)}$, the fusion of conjugacy classes of $P_{2}$ into $L_{4}(3)$ and the restrictions of $\chi_{i} \in \operatorname{Irr}\left(L_{4}(3)\right)$ to $P_{2}$, where $\operatorname{deg}\left(\chi_{i}\right)<234$ are used. Thus restricting $\chi_{i} \in \operatorname{Irr}\left(L_{4}(3)\right)$ to $P_{2}$ where $i \in 1,2,3, \ldots, 8$, and computing the inner product $\left\langle\chi_{i}, \psi_{1}\right\rangle$ of each $\chi_{i}, i \in 1,2,3, \ldots, 8$, with the identity character $\psi_{1}$ of $P_{2}$, the values below are obtained.

\begin{tabular}{|c|c|c|c|c|c|c|c|c|}
\hline & $\chi_{1}$ & $\chi_{2}$ & $\chi_{3}$ & $\chi_{4}$ & $\chi_{5}$ & $\chi_{6}$ & $\chi_{7}$ & $\chi_{8}$ \\
\hline$\left\langle\chi_{i}, \psi_{1}\right\rangle$ & 1 & 0 & 1 & 0 & 1 & 0 & 1 & 1 \\
\hline
\end{tabular}

From the above table and taking into consideration the FrobeniusReciprocity theorem [12], the permutation character $I_{P_{2}}^{L_{4}(3)}=1 a+26 b+$ $52 a+65 b+90 a$ is obtained. Similarly, $I_{P_{4}}^{L_{4}(3)}$ is determined. In this case, we restrict $\chi_{i} \in \operatorname{Irr}\left(L_{4}(3)\right.$, where $i \in 1,2,3, \ldots, 10$ to $P_{4}$ and let $\psi_{1}$ be the identity character $P_{4}$. Computing the inner product $\left\langle\chi_{i}, \psi_{1}\right\rangle$ of each $\chi_{i}$ for $i \in 1,2,3, \ldots, 10$ with $\psi_{1}$, the values below are found.

\begin{tabular}{|c|c|c|c|c|c|c|c|c|c|c|}
\hline & $\chi_{1}$ & $\chi_{2}$ & $\chi_{3}$ & $\chi_{4}$ & $\chi_{5}$ & $\chi_{6}$ & $\chi_{7}$ & $\chi_{8}$ & $\chi_{9}$ & $\chi_{10}$ \\
\hline$\left\langle\chi_{i}, \psi_{1}\right\rangle$ & 1 & 0 & 0 & 1 & 0 & 1 & 1 & 1 & 0 & 0 \\
\hline
\end{tabular}

The identity character $I_{P_{4}}^{L_{4}(3)}$ of $P_{4}$ induced to $L_{4}(3)$ is therefore given by, $I_{P_{4}}^{L_{4}(3)}=1 a+39 a+65 a+65 b+90 a$. It follows that the permutation character $\chi\left(L_{4}(3) \mid 3^{6}\right)$ is given as,

$$
\begin{aligned}
\chi\left(L_{4}(3) \mid 3^{6}\right) & =1+2 I_{P_{2}}^{L_{4}(3)}+I_{P_{4}}^{L_{4}(3)} \\
& =1 a+2(1 a+26 b+52 a+65 b+90 a)+1 a+39 a+65 a+65 b+90 a \\
& =4 \times 1 a+2 \times 26 b+39 a+2 \times 52 a+65 a+3 \times 65 b+3 \times 90 a .
\end{aligned}
$$

The permutation characters $\chi\left(L_{4}(3) \mid P_{i}\right)$ are written in terms of the ordinary irreducible characters of $G$ and are computed directly using the character table of $G$. The permutation character $\chi\left(L_{4}(3) \mid 3^{6}\right)$ on the different conjugacy classes of $G$ determines the number $k$ of fixed points of each $g \in G$ in $3^{6}$. The values of $k$ obtained by the above permutation character are listed in Table 1. 
Table 1: Permutation Character of $G=L_{4}(3)$ on $3^{6}$

\begin{tabular}{|c|c|c|c|c|c|c|c|c|c|c|c|c|c|c|c|}
\hline $9 \mid G$ & $1 \mathrm{~A}$ & $2 A$ & $2 B$ & $3 A$ & $3 B$ & $3 C$ & $3 D$ & $4 A$ & $4 B$ & $4 C$ & $5 A$ & $6 A$ & $6 B$ & 60 & $6 D$ \\
\hline$\chi\left(G \mid P_{1}\right)$ & 1 & 1 & 1 & 1 & 1 & 1 & 1 & 1 & 1 & 1 & 1 & 1 & 1 & 1 & 1 \\
\hline$\chi\left(G \mid P_{2}\right)$ & 234 & 30 & 2 & 18 & 0 & 18 & 36 & 0 & 2 & 4 & 4 & 0 & 6 & 2 & 2 \\
\hline$\chi\left(G \mid P_{3}\right)$ & 234 & 30 & 2 & 18 & 0 & 18 & 36 & 0 & 2 & 4 & 4 & 0 & 6 & 2 & 2 \\
\hline$\chi\left(G \mid P_{4}\right)$ & 260 & 20 & 4 & 44 & 8 & 26 & 26 & 0 & 4 & 0 & 0 & 2 & 2 & 4 & 4 \\
\hline$k$ & 729 & 81 & 9 & 81 & 9 & 63 & 99 & 1 & 9 & 9 & 9 & 3 & 15 & 9 & 9 \\
\hline & $6 E$ & $8 A$ & $9 A$ & $9 B$ & $10 \mathrm{~A}$ & $12 \mathrm{~A}$ & $12 B$ & $12 \mathrm{C}$ & $13 \mathrm{~A}$ & $13 B$ & $13 \mathrm{C}$ & $13 D$ & $20 \mathrm{~A}$ & $20 B$ & \\
\hline$\chi\left(G \mid P_{1}\right)$ & 1 & 1 & 1 & 1 & 1 & 1 & 1 & 1 & 1 & 1 & 1 & 1 & 1 & 1 & \\
\hline$\chi\left(G \mid P_{2}\right)$ & 2 & 0 & 0 & 6 & 0 & 0 & 0 & 2 & 0 & 0 & 0 & 0 & 0 & 0 & \\
\hline$\chi\left(G \mid P_{S}\right)$ & 2 & 0 & 0 & 6 & 0 & 0 & 0 & 2 & 0 & 0 & 0 & 0 & 0 & 0 & \\
\hline$x\left(G \mid P_{4}\right)$ & 4 & 0 & 2 & 2 & 0 & 0 & 0 & 4 & 0 & 0 & 0 & 0 & 0 & 0 & \\
\hline$k$ & 9 & 1 & 3 & 15 & 1 & 1 & 1 & 9 & 1 & 1 & 1 & 1 & 1 & 1 & \\
\hline
\end{tabular}

Clearly, $\chi\left(L_{4}(3) \mid 3^{6}\right)=4 \times 1 a+2 \times 26 b+39 a+2 \times 52 a+65 a+3 \times$ $65 b+3 \times 90 a$ is not the required permutation character of $G$ acting on $N$ since $k \neq 3^{n}, n \in\{0,1,2,3,4,5,6\}$ for all the conjugacy classes of $G$. In order to obtain values of $k$ such that $k=3^{n}, n \in\{0,1,2,3,4,5,6\}$, another possible fusion of conjugacy classes of $P_{2} \cong P_{3}$ into $L_{4}(3)$ is considered. We restrict $\chi_{i} \in \operatorname{Irr}\left(L_{4}(3)\right)$ to $P_{2}$, and then compute the inner product $\left\langle\chi_{i}, \psi_{1}\right\rangle$ of each $\chi_{i}, i \in 1,2,3, \ldots, 8$ with the identity character $\psi_{1}$ of $P_{2}$. The values of $\left\langle\chi_{i}, \psi_{1}\right\rangle$ are listed below,

\begin{tabular}{|c|c|c|c|c|c|c|c|c|}
\hline & $\chi_{1}$ & $\chi_{2}$ & $\chi_{3}$ & $\chi_{4}$ & $\chi_{5}$ & $\chi_{6}$ & $\chi_{7}$ & $\chi_{8}$ \\
\hline$\left\langle\chi_{i}, \psi_{1}\right\rangle$ & 1 & 1 & 0 & 0 & 1 & 1 & 0 & 1 \\
\hline
\end{tabular}

From the above table and taking into consideration the FrobeniusReciprocity theorem, the permutation character $I_{P_{2}}^{L_{4}(3)}$ now assumes the following form,

$$
I_{P_{2}}^{L_{4}(3)}=1 a+26 a+52 a+65 a+90 a .
$$

Using $I_{P_{2}}^{L_{4}(3)}=1 a+26 a+52 a+65 a+90 a$ and $I_{P_{3}}^{L_{4}(3)}=1 a+26 b+52 a+$ $65 b+90 a$ we obtain that

$$
\begin{aligned}
\chi\left(L_{4}(3) \mid 3^{6}\right)= & 1+I_{P_{2}}^{L_{4}(3)}+I_{P_{3}}^{L_{4}(3)}+I_{P_{4}}^{L_{4}(3)} \\
= & 4 \times 1 a+1 \times 26 a+1 \times 26 b+1 \times 39 a+2 \times 52 a \\
& +2 \times 65 a+2 \times 65 b+3 \times 90 a .
\end{aligned}
$$


Using this result, the correct number $k$ of fixed points of each $g \in G$ in $3^{6}$ is obtained and is listed in Table 2. This confirms that $P_{2}$ and $P_{3}$ sit separately inside the two maximal subgroups of $G$ of the form $U_{4}(2): 2$.

Table 2: Permutation Character of $G=L_{4}(3)$ on $3^{6}$

\begin{tabular}{|c|c|c|c|c|c|c|c|c|c|c|c|c|c|c|c|}
\hline$|9|_{G}$ & $1 \mathrm{~A}$ & $2 A$ & $2 B$ & $3 . A$ & $3 B$ & $3 C$ & $3 D$ & $4 A$ & $4 B$ & $4 C$ & $5 A$ & $6 A$ & $6 B$ & $6 C$ & $6 D$ \\
\hline$\chi\left(G \mid P_{1}\right)$ & 1 & 1 & 1 & 1 & 1 & 1 & 1 & 1 & 1 & 1 & 1 & 1 & 1 & 1 & 1 \\
\hline$\chi\left(G \mid P_{2}\right)$ & 234 & 30 & 2 & 18 & 0 & 18 & 96 & 0 & 2 & 4 & 4 & 0 & 6 & 2 & 2 \\
\hline$\chi\left(G \mid P_{3}\right)$ & 234 & 30 & 2 & 18 & 0 & 36 & 18 & 0 & 2 & 4 & 4 & 6 & 0 & 2 & 2 \\
\hline$\chi\left(G \mid P_{4}\right)$ & 260 & 20 & 4 & 44 & 8 & 26 & 26 & 0 & 4 & 0 & 0 & 2 & 2 & 4 & 4 \\
\hline$k$ & 729 & 81 & 9 & 81 & 9 & 81 & 81 & 1 & 9 & 9 & 9 & 9 & 9 & 9 & 9 \\
\hline & $6 E$ & $8 A$ & $9 A$ & $9 B$ & $10 \mathrm{~A}$ & $12 \mathrm{~A}$ & $12 B$ & $12 \mathrm{C}$ & $13 \mathrm{~A}$ & $13 B$ & $13 C$ & $13 D$ & $20 \mathrm{~A}$ & $20 B$ & \\
\hline$\chi\left(G \mid P_{1}\right)$ & 1 & 1 & 1 & 1 & 1 & 1 & 1 & 1 & 1 & 1 & 1 & 1 & 1 & 1 & \\
\hline$\chi\left(G \mid P_{2}\right)$ & 2 & 0 & 0 & 6 & 0 & 0 & 0 & 2 & 0 & 0 & 0 & 0 & 0 & 0 & \\
\hline$\chi\left(G \mid P_{S}\right)$ & 2 & 0 & 6 & 0 & 0 & 0 & 0 & 2 & 0 & 0 & 0 & 0 & 0 & 0 & \\
\hline$\chi\left(G \mid P_{4}\right)$ & 4 & 0 & 2 & 2 & 0 & 0 & 0 & 4 & 0 & 0 & 0 & 0 & 0 & 0 & \\
\hline$k$ & 9 & 1 & 9 & 9 & 1 & 1 & 1 & 9 & 1 & 1 & 1 & 1 & 1 & 1 & \\
\hline
\end{tabular}

\subsection{The Classes of $\bar{G}=3^{6}: L_{4}(3)$}

The values of $k$ enable us to determine the number $f_{j}$ of orbits $Q_{i}$ 's, $1 \leq$ $i \leq k$ that fuse together under the action of $C_{G}(g)$ to form one orbit $\triangle_{j}$ (see [18]). To determine the values of these $f_{j}$ 's and the orders of class representatives $d g \in \bar{G}$, Programmes $\mathrm{A}$ and $\mathrm{B}$ in [24] written in GAP are used, respectively. If $o(g)=m$ and $w=1_{N}$ then $o(d g)=m$ and if $w \neq 1_{N}$ then $o(d g)=3 m$ (see Theorem 2.3.10 in [18]). The formula $\left|C_{\bar{G}}(x)\right|=\frac{k}{f_{j}}\left|C_{G}(g)\right|$ is then used to calculate the order of the centralizer of each class of $\bar{G}$ with representative $x$ and a constant $m_{j}=\frac{f_{j}}{k}|N|$ is also calculated for each value of $f_{j}$. This constant plays a very crucial role in determination of the entries of the Fischer Clifford matrices. The group $\bar{G}=3^{6}: L_{4}(3)$ is found to have 111 conjugacy classes of elements. Table 3 below gives a detailed information on the conjugacy classes of $\bar{G}=3^{6}: L_{4}(3)$. The power maps of elements of $\bar{G}$ are given in the second last column of Table 3 whereas the fusion of $\bar{G}$ into $O_{8}^{+}(3)$, as determined in Section 8 of this paper, is found in the last column of Table 3. 
Table 3: The Congujacy Classes of $\bar{G}=3^{6}: L_{4}(3)$

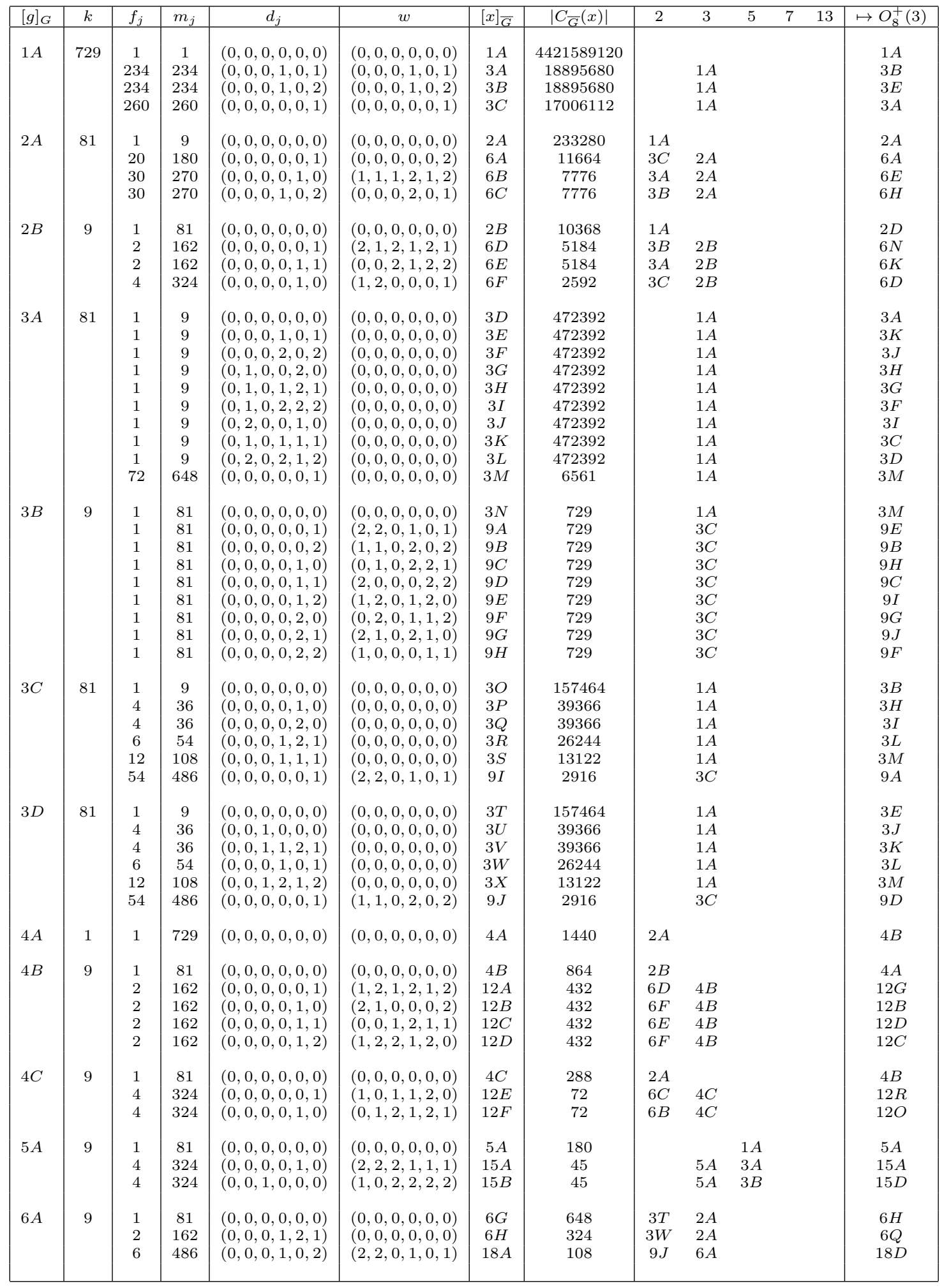


Table 3: (continued)

\begin{tabular}{|c|c|c|c|c|c|c|c|c|c|c|c|c|c|}
\hline$[g]_{G}$ & $k$ & $f_{j}$ & $m_{j}$ & $d_{j}$ & $w$ & {$[x]_{\bar{G}}$} & $\left|C_{\bar{G}}(x)\right|$ & 2 & 3 & 5 & 7 & 13 & $\mapsto O_{8}^{+}(3)$ \\
\hline \multirow[t]{3}{*}{$6 B$} & 9 & 1 & 81 & $(0,0,0,0,0,0)$ & $(0,0,0,0,0,0)$ & $6 I$ & 648 & $3 O$ & $2 A$ & & & & $6 E$ \\
\hline & & 2 & 162 & $(0,0,0,1,1,1)$ & $(0,0,0,0,0,0)$ & $6 J$ & 324 & $3 R$ & $2 A$ & & & & $6 Q$ \\
\hline & & 6 & 486 & $(0,1,0,0,1,0)$ & $(1,1,0,2,0,2)$ & $18 B$ & 108 & $9 I$ & $6 A$ & & & & $18 \mathrm{~A}$ \\
\hline \multirow[t]{9}{*}{$6 C$} & 9 & 1 & 81 & $(0,0,0,0,0,0)$ & $(0,0,0,0,0,0)$ & $6 K$ & 648 & $3 D$ & $2 B$ & & & & $6 D$ \\
\hline & & 1 & 81 & $(0,0,0,0,0,1)$ & $(0,0,0,0,0,0)$ & $6 L$ & 648 & $3 F$ & $2 B$ & & & & $6 \mathrm{~V}$ \\
\hline & & 1 & 81 & $(0,0,0,0,0,2)$ & $(0,0,0,0,0,0)$ & $6 M$ & 648 & $3 E$ & $2 B$ & & & & $6 W$ \\
\hline & & 1 & 81 & $(0,0,0,0,1,0)$ & $(0,0,0,0,0,0)$ & $6 N$ & 648 & $3 K$ & $2 B$ & & & & $6 L$ \\
\hline & & 1 & 81 & $(0,0,0,0,1,1)$ & $(0,0,0,0,0,0)$ & $6 O$ & 648 & $3 J$ & $2 B$ & & & & $6 U$ \\
\hline & & 1 & 81 & $(0,0,0,0,1,2)$ & $(0,0,0,0,0,0)$ & $6 P$ & 648 & $3 L$ & $2 B$ & & & & $6 M$ \\
\hline & & 1 & 81 & $(0,0,0,0,2,0)$ & $(0,0,0,0,0,0)$ & $6 Q$ & 648 & $3 I$ & $2 B$ & & & & $6 O$ \\
\hline & & 1 & 81 & $(0,0,0,0,2,1)$ & $(0,0,0,0,0,0)$ & $6 R$ & 648 & $3 H$ & $2 B$ & & & & $6 P$ \\
\hline & & 1 & 81 & $(0,0,0,0,2,2)$ & $(0,0,0,0,0,0)$ & $6 S$ & 648 & $3 G$ & $2 B$ & & & & $6 T$ \\
\hline \multirow[t]{6}{*}{$6 D$} & 9 & 1 & 81 & $(0,0,0,0,0,0)$ & $(0,0,0,0,0,0)$ & $6 T$ & 324 & $3 O$ & $2 B$ & & & & $6 K$ \\
\hline & & 1 & 81 & $(0,0,0,0,1,1)$ & $(0,0,0,0,0,0)$ & $6 U$ & 324 & $3 R$ & $2 B$ & & & & $6 Y$ \\
\hline & & 1 & 81 & $(0,0,0,0,2,2)$ & $(0,0,0,0,0,0)$ & $6 \mathrm{~V}$ & 324 & $3 R$ & $2 B$ & & & & $6 X$ \\
\hline & & 2 & 162 & $(0,0,0,0,0,1)$ & $(0,0,0,0,0,0)$ & $6 W$ & 162 & $3 P$ & $2 B$ & & & & $6 T$ \\
\hline & & 2 & 162 & $(0,0,0,0,1,0)$ & $(0,0,0,0,0,0)$ & $6 X$ & 162 & $3 Q$ & $2 B$ & & & & $6 U$ \\
\hline & & 2 & 162 & $(0,0,0,0,2,0)$ & $(0,0,0,0,0,0)$ & $6 Y$ & 162 & $3 S$ & $2 B$ & & & & $6 A B$ \\
\hline \multirow[t]{6}{*}{$6 E$} & 9 & 1 & 81 & $(0,0,0,0,0,0)$ & $(0,0,0,0,0,0)$ & $6 Z$ & 324 & $3 T$ & $2 B$ & & & & $6 N$ \\
\hline & & 1 & 81 & $(0,0,0,0,1,1)$ & $(0,0,0,0,0,0)$ & $6 A A$ & 324 & $3 W$ & $2 B$ & & & & $6 A A$ \\
\hline & & 1 & 81 & $(0,0,0,0,2,2)$ & $(0,0,0,0,0,0)$ & $6 A B$ & 324 & $3 W$ & $2 B$ & & & & $6 Z$ \\
\hline & & 2 & 162 & $(0,0,0,0,0,1)$ & $(0,0,0,0,0,0)$ & $6 A C$ & 162 & $3 U$ & $2 B$ & & & & $6 \mathrm{~V}$ \\
\hline & & 2 & 162 & $(0,0,0,0,1,0)$ & $(0,0,0,0,0,0)$ & $6 A D$ & 162 & $3 \mathrm{~V}$ & $2 B$ & & & & $6 W$ \\
\hline & & 2 & 162 & $(0,0,0,0,2,0)$ & $(0,0,0,0,0,0)$ & $6 A E$ & 162 & $3 X$ & $2 B$ & & & & $6 A B$ \\
\hline $8 A$ & 1 & 1 & 729 & $(0,0,0,0,0,0)$ & $(0,0,0,0,0,0)$ & $8 A$ & 8 & $4 B$ & & & & & $8 A$ \\
\hline \multirow[t]{5}{*}{$9 A$} & 9 & 1 & 81 & $(0,0,0,0,0,0)$ & $(0,0,0,0,0,0)$ & $9 K$ & 243 & & $3 D$ & & & & $9 A$ \\
\hline & & 1 & 81 & $(0,0,0,0,1,1)$ & $(0,0,0,0,0,0)$ & $9 L$ & 243 & & $3 D$ & & & & $9 T$ \\
\hline & & 1 & 81 & $(0,1,0,0,2,0)$ & $(0,0,0,0,0,0)$ & $9 M$ & 243 & & $3 D$ & & & & $9 J$ \\
\hline & & 3 & 243 & $(0,0,0,0,0,1)$ & $(0,0,0,0,0,0)$ & $9 N$ & 81 & & $3 J$ & & & & $9 L$ \\
\hline & & 3 & 243 & $(0,0,0,0,0,2)$ & $(0,0,0,0,0,0)$ & $9 O$ & 81 & & $3 G$ & & & & $9 K$ \\
\hline \multirow[t]{5}{*}{$9 B$} & 9 & 1 & 81 & $(0,0,0,0,0,0)$ & $(0,0,0,0,0,0)$ & $9 P$ & 243 & & $3 D$ & & & & $9 D$ \\
\hline & & 1 & 81 & $(1,0,0,0,1,1)$ & $(0,0,0,0,0,0)$ & $9 Q$ & 243 & & $3 D$ & & & & $9 H$ \\
\hline & & 1 & 81 & $(2,0,0,0,2,0)$ & $(0,0,0,0,0,0)$ & $9 R$ & 243 & & $3 D$ & & & & $9 G$ \\
\hline & & 3 & 243 & $(0,0,0,0,1,0)$ & $(0,0,0,0,0,0)$ & $9 S$ & 81 & & $3 E$ & & & & $9 N$ \\
\hline & & 3 & 243 & $(0,0,0,0,2,0)$ & $(0,0,0,0,0,0)$ & $9 T$ & 81 & & $3 F$ & & & & $9 M$ \\
\hline $10 A$ & 1 & 1 & 729 & $(0,0,0,0,0,0)$ & $(0,0,0,0,0,0)$ & $10 A$ & 20 & $5 A$ & & & & & $10 A$ \\
\hline $12 A$ & 1 & 1 & 729 & $(0,0,0,0,0,0)$ & $(0,0,0,0,0,0)$ & $12 G$ & 36 & $6 G$ & $4 A$ & & & & $12 R$ \\
\hline $12 B$ & 1 & 1 & 729 & $(0,0,0,0,0,0)$ & $(0,0,0,0,0,0)$ & $12 \mathrm{H}$ & 36 & $6 I$ & $4 A$ & & & & $12 \mathrm{O}$ \\
\hline \multirow[t]{9}{*}{$12 C$} & 9 & 1 & 81 & $(0,0,0,0,0,0)$ & $(0,0,0,0,0,0)$ & $12 I$ & 108 & $6 K$ & $4 B$ & & & & $12 A$ \\
\hline & & 1 & 81 & $(0,0,0,0,0,1)$ & $(0,0,0,0,0,0)$ & $12 J$ & 108 & $6 M$ & $4 B$ & & & & $12 N$ \\
\hline & & 1 & 81 & $(0,0,0,0,0,2)$ & $(0,0,0,0,0,0)$ & $12 K$ & 108 & $6 L$ & $4 B$ & & & & $12 M$ \\
\hline & & 1 & 81 & $(0,0,0,1,0,0)$ & $(0,0,0,0,0,0)$ & $12 L$ & 108 & $6 Q$ & $4 B$ & & & & $12 H$ \\
\hline & & 1 & 81 & $(0,0,0,1,0,1)$ & $(0,0,0,0,0,0)$ & $12 M$ & 108 & $6 S$ & $4 B$ & & & & $12 K$ \\
\hline & & 1 & 81 & $(0,0,0,1,0,2)$ & $(0,0,0,0,0,0)$ & $12 N$ & 108 & $6 R$ & $4 B$ & & & & $12 I$ \\
\hline & & 1 & 81 & $(0,0,0,2,0,0)$ & $(0,0,0,0,0,0)$ & $12 O$ & 108 & $6 N$ & $4 B$ & & & & $12 E$ \\
\hline & & 1 & 81 & $(0,0,0,2,0,1)$ & $(0,0,0,0,0,0)$ & $12 P$ & 108 & $6 P$ & $4 B$ & & & & $12 F$ \\
\hline & & 1 & 81 & $(0,0,0,2,0,2)$ & $(0,0,0,0,0,0)$ & $12 Q$ & 108 & $6 O$ & $4 B$ & & & & $12 L$ \\
\hline $13 A$ & 1 & 1 & 729 & $(0,0,0,0,0,0)$ & $(0,0,0,0,0,0)$ & $13 \mathrm{~A}$ & 13 & $13 D$ & & $13 D$ & & $1 A$ & $13 B$ \\
\hline $13 B$ & 1 & 1 & 729 & $(0,0,0,0,0,0)$ & $(0,0,0,0,0,0)$ & $13 B$ & 13 & $13 \mathrm{~A}$ & & $13 A$ & & $1 \mathrm{~A}$ & $13 \mathrm{~A}$ \\
\hline $13 C$ & 1 & 1 & 729 & $(0,0,0,0,0,0)$ & $(0,0,0,0,0,0)$ & $13 C$ & 13 & $13 B$ & & $13 B$ & & $1 \mathrm{~A}$ & $13 B$ \\
\hline $13 D$ & 1 & 1 & 729 & $(0,0,0,0,0,0)$ & $(0,0,0,0,0,0)$ & $13 D$ & 13 & $13 C$ & & $13 C$ & & $1 \mathrm{~A}$ & $13 \mathrm{~A}$ \\
\hline $20 A$ & 1 & 1 & 729 & $(0,0,0,0,0,0)$ & $(0,0,0,0,0,0)$ & $20 A$ & 20 & $10 \mathrm{~A}$ & & $4 A$ & & & $20 A$ \\
\hline $20 B$ & 1 & 1 & 729 & $(0,0,0,0,0,0)$ & $(0,0,0,0,0,0)$ & $20 B$ & 20 & $10 \mathrm{~A}$ & & $4 A$ & & & $20 A$ \\
\hline
\end{tabular}




\section{Inertia Factor Groups of $\bar{G}=3^{6}: L_{4}(3)$}

We have already seen in Section 3 that the action of $G=L_{4}(3)$ on $N=3^{6}$ has four orbits of lengths 1, 234, 234 and 260. By Brauer's theorem (see Theorem 5.1.5 in [18]), the action of $G$ on $\operatorname{Irr}(N)$ will also have four orbits of lengths $1, r, s$ and $t$ with $1+r+s+t=729$ such that $\left[G: H_{1}\right]=1,\left[G: H_{2}\right]=$ $r,\left[G: H_{3}\right]=s$ and $\left[G: H_{4}\right]=t$, where $H_{1}, H_{2}, H_{3}$ and $H_{4}$ are the inertia factor groups of $\bar{G}$. When $N$ is an elementary abelian $p$-group, then it can be regarded as a vector space $V$ over $F=G F(p)$. Any vector space has a dual (the set of all linear functional maps from $V$ into $F$ ) denoted by $V^{*}$. Although $V$ and $V^{*}$ are isomorphic as vector spaces (so they have the same dimension), they may not be equivalent as $G$-modules. It is easy to show that $N^{*}=\operatorname{Irr}(N)$ and hence the action of $G$ on $\operatorname{Irr}(N)$ is the same as action of $G$ on $N^{*}$. Seretlo [24] developed a programme for the action of $G$ on $V^{*}$. It is found in the Brauer ATLAS that $G \cong O_{6}^{+}(3)$ has only one irreducible module of dimension 6 over $G F(3)$ and thus $N$ and $N^{*}$ are equivalent as $G$-modules. Hence the actions of $G$ on $N$ and $N^{*}$ are isomorphic, and so the point stabilizers $P_{i}$ and stabilizers on $N^{*}$ (inertia factor groups) are the same. We now have that $r=234, s=234$ and $t=260$ and that $H_{1}=L_{4}(3), H_{2} \cong H_{3}=U_{4}(2)$ and $H_{4}=3^{4}: 2\left(A_{4} \times A_{4}\right)$. Using GAP, the number of conjugacy classes of the inertia factor groups are determined and it turns out that

$$
\left|\operatorname{Irr}\left(H_{1}\right)\right|+\left|\operatorname{Irr}\left(H_{2}\right)\right|+\left|\operatorname{Irr}\left(H_{3}\right)\right|+\left|\operatorname{Irr}\left(H_{4}\right)\right|=29+20+20+42=111 .
$$

This shows that the total contribution of irreducible characters from the four inertia groups is 111 and is equal to the number of classes of $\bar{G}$ as determined in Section 3. The inertia factor groups of $\bar{G}$ are constructed from elements within $G=L_{4}(3)$ and their generators are as follows:

$H_{2}=U_{4}(2)=\left\langle\alpha_{1}, \alpha_{2}\right\rangle, \alpha_{1} \in 6 A, \alpha_{2} \in 4 B$, where

$$
\alpha_{1}=\left(\begin{array}{cccccc}
1 & 0 & 1 & 0 & 2 & 0 \\
1 & 0 & 0 & 0 & 0 & 2 \\
2 & 0 & 0 & 2 & 2 & 2 \\
0 & 0 & 0 & 1 & 0 & 0 \\
1 & 1 & 0 & 1 & 2 & 1 \\
0 & 0 & 0 & 0 & 0 & 1
\end{array}\right), \quad \alpha_{2}=\left(\begin{array}{cccccc}
2 & 2 & 0 & 1 & 0 & 1 \\
1 & 0 & 1 & 2 & 0 & 1 \\
2 & 2 & 2 & 2 & 1 & 2 \\
2 & 2 & 1 & 0 & 0 & 1 \\
2 & 0 & 2 & 2 & 0 & 2 \\
0 & 0 & 0 & 0 & 0 & 1
\end{array}\right)
$$


$H_{3}=U_{4}(2)=\left\langle\beta_{1}, \beta_{2}\right\rangle, \beta_{1} \in 6 B, \beta_{2} \in 4 C$, where

$$
\beta_{1}=\left(\begin{array}{cccccc}
2 & 2 & 2 & 2 & 0 & 0 \\
1 & 2 & 2 & 1 & 0 & 1 \\
0 & 1 & 0 & 2 & 1 & 0 \\
0 & 1 & 1 & 1 & 2 & 1 \\
0 & 2 & 2 & 0 & 2 & 2 \\
2 & 0 & 1 & 1 & 2 & 1
\end{array}\right), \quad \beta_{2}=\left(\begin{array}{llllll}
2 & 2 & 1 & 0 & 1 & 1 \\
1 & 2 & 2 & 0 & 1 & 1 \\
2 & 0 & 0 & 1 & 0 & 2 \\
2 & 2 & 2 & 1 & 0 & 1 \\
1 & 1 & 1 & 0 & 1 & 2 \\
0 & 2 & 1 & 1 & 2 & 0
\end{array}\right)
$$

$H_{4}=3^{4}: 2\left(A_{4} \times A_{4}\right)=\left\langle\gamma_{1}, \gamma_{2}, \gamma_{3}\right\rangle, \gamma_{1}, \gamma_{2}, \gamma_{3} \in 2 A$, where

$$
\begin{gathered}
\gamma_{1}=\left(\begin{array}{llllll}
1 & 0 & 0 & 0 & 0 & 0 \\
0 & 0 & 1 & 0 & 0 & 0 \\
0 & 1 & 0 & 0 & 0 & 0 \\
2 & 1 & 1 & 0 & 2 & 1 \\
1 & 2 & 2 & 1 & 2 & 2 \\
2 & 1 & 1 & 2 & 2 & 2
\end{array}\right), \quad \gamma_{2}=\left(\begin{array}{llllll}
1 & 0 & 0 & 0 & 0 & 0 \\
0 & 0 & 1 & 0 & 0 & 0 \\
0 & 1 & 0 & 0 & 0 & 0 \\
2 & 1 & 1 & 0 & 2 & 1 \\
1 & 2 & 2 & 1 & 2 & 2 \\
2 & 1 & 1 & 2 & 2 & 2
\end{array}\right), \\
\gamma_{3}=\left(\begin{array}{llllll}
1 & 0 & 0 & 0 & 0 & 0 \\
0 & 0 & 1 & 0 & 0 & 0 \\
0 & 1 & 0 & 0 & 0 & 0 \\
2 & 1 & 1 & 0 & 2 & 1 \\
1 & 2 & 2 & 1 & 2 & 2 \\
2 & 1 & 1 & 2 & 2 & 2
\end{array}\right)
\end{gathered}
$$

\section{The fusion of $H_{2}, H_{3}$ and $H_{4}$ into $G=L_{4}(3)$}

The fusion maps of the inertia factor groups $H_{2}, H_{3}$ and $H_{4}$ into $G=L_{4}(3)$ are obtained, by using the generators of the $H_{i}$ 's and the GAP command "FusionConjugacyClasses $\left(H_{i}, G\right)$ ". The complete fusion maps of $H_{2}, H_{3}$ and $H_{4}$ into $G=L_{4}(3)$ are shown in Tables 4,5 and 6 below. 
Table 4: The fusion of $H_{2}$ into $G$

\begin{tabular}{|c|c|c|c|c|c|c|c|}
\hline$[h]_{H}$ & {$[g]_{L_{4}(3)}$} & {$[h]_{H}$} & {$[g]_{L_{4}(3)}$} & {$[h]_{H}$} & {$[g]_{L_{4}(3)}$} & {$[h]_{H_{2}}$} & {$[g]_{L_{4}(3)}$} \\
\hline $1 A$ & $1 A$ & $3 C$ & $3 D$ & $6 A$ & $6 C$ & $6 F$ & $6 B$ \\
\hline $2 A$ & $2 B$ & $3 D$ & $3 C$ & $6 B$ & $6 C$ & $9 A$ & $9 B$ \\
\hline $2 B$ & $2 A$ & $4 A$ & $4 B$ & $6 C$ & $6 E$ & $9 B$ & $9 B$ \\
\hline $3 A$ & $3 A$ & $4 B$ & $4 C$ & $6 D$ & $6 D$ & $12 A$ & $12 C$ \\
\hline $3 B$ & $3 A$ & $5 A$ & $5 A$ & $6 E$ & $6 D$ & $12 B$ & $12 C$ \\
\hline
\end{tabular}

Table 5: The fusion of $H_{3}$ into $G$

\begin{tabular}{|ll||lc||lc||cc|}
\hline$[h]_{H_{3}} \longrightarrow[g]_{L_{4}(3)}$ & {$[h]_{H_{3}} \longrightarrow[g]_{L_{4}(3)}$} & {$[h]_{H_{3}} \longrightarrow[g]_{L_{4}(3)}$} & {$[h]_{H_{4}} \longrightarrow[g]_{L_{4}(3)}$} \\
\hline $1 A$ & $1 A$ & $3 C$ & $3 A$ & $6 A$ & $6 D$ & $6 F$ & $6 A$ \\
$2 A$ & $2 B$ & $3 D$ & $3 D$ & $6 B$ & $6 C$ & $9 A$ & $9 A$ \\
$2 B$ & $2 A$ & $4 A$ & $4 B$ & $6 C$ & $6 C$ & $9 B$ & $9 A$ \\
$3 A$ & $3 C$ & $4 B$ & $4 C$ & $6 D$ & $6 E$ & $12 A$ & $12 C$ \\
$3 B$ & $3 A$ & $5 A$ & $5 A$ & $6 E$ & $6 E$ & $12 B$ & $12 C$ \\
\hline
\end{tabular}

Table 6: The fusion of $H_{4}$ into $G$

\begin{tabular}{|ll||lc||lc||cc|}
\hline$[h]_{H_{4}} \longrightarrow[g]_{L_{4}(3)}$ & {$[h]_{H_{4}} \longrightarrow[g]_{L_{4}}(3)$} & {$[h]_{H_{4}} \longrightarrow[g]_{L_{4}(3)}$} & {$[h]_{H_{4}} \longrightarrow[g]_{L_{4}(3)}$} \\
\hline $1 A$ & $1 A$ & $3 I$ & $3 B$ & $4 A$ & $4 B$ & $6 I$ & $6 C$ \\
$2 A$ & $2 B$ & $3 J$ & $3 C$ & $4 B$ & $4 B$ & $6 J$ & $6 D$ \\
$2 B$ & $2 A$ & $3 K$ & $3 B$ & $6 A$ & $6 A$ & $9 A$ & $9 B$ \\
$3 A$ & $3 C$ & $3 L$ & $3 A$ & $6 B$ & $6 B$ & $9 B$ & $9 A C$ \\
$3 B$ & $3 A$ & $3 M$ & $3 B$ & $6 C$ & $6 C$ & $9 C$ & $9 B$ \\
$3 C$ & $3 D$ & $3 N$ & $3 D$ & $6 D$ & $6 C$ & $9 D$ & $9 A$ \\
$3 D$ & $3 A$ & $3 O$ & $3 B$ & $6 E$ & $6 E$ & $12 A$ & $12 C$ \\
$3 E$ & $3 B$ & $3 P$ & $3 A$ & $6 F$ & $6 D$ & $12 B$ & $12 C$ \\
$3 F$ & $3 A$ & $3 Q$ & $3 B$ & $6 G$ & $6 C$ & $12 C$ & $12 C$ \\
$3 G$ & $3 B$ & $3 R$ & $3 C$ & $6 H$ & $6 E$ & $12 D$ & $12 C$ \\
$3 H$ & $3 D$ & $3 S$ & $3 B$ & & & & \\
\hline
\end{tabular}




\section{The Fischer-Matrices of $\bar{G}=3^{6}: L_{4}(3)$}

Having obtained the conjugacy classes of $\bar{G}$ in coset-analysis format and the fusion maps of the inertia factor groups $H_{2}, H_{3}$ and $H_{4}$ into $G$, the Fischer-Clifford matrices of the group $\bar{G}=3^{6}: L_{4}(3)$ will be now computed. Programme $\mathrm{D}$ in [5] is largely used for automatic determination of a possible candidate for each Fischer-Clifford matrix $M(g), g \in G$, of $\bar{G}$. Then the properties of Fischer-Clifford matrices discussed in detail in [6], [13], [14] and [23] are used to rearrange the rows and columns of this candidate in order to get the unique matrix $M(g)$ for $\bar{G}=3^{6}: L_{4}(3)$. The Programme D only works on split extensions $N: G$, where $N$ is elementary abelian. Note that since $N=3^{6}$ is an elementary abelian $p$-group, then all the relations hold. For example, considering the conjugacy class $2 A$ of $G=L_{4}(3)$, and by making use of Theorem 5.2.4 and property (e) in [18], $M(2 A)$ has the following form with corresponding weights attached to the rows and columns.

$$
\begin{aligned}
& \left|C_{\bar{G}}(2 A)\right| \quad\left|C_{\bar{G}}(6 A)\right| \quad\left|C_{\bar{G}}(6 B)\right| \quad\left|C_{\bar{G}}(6 C)\right| \\
& \begin{array}{llll}
233280 & 11664 & 7776 & 7776
\end{array} \\
& \begin{aligned}
\left|C_{H_{1}}(2 A)\right|= & 2880 \\
\left|C_{H_{2}}(2 B)\right|= & 96 \\
\left|C_{H_{3}}(2 B)\right|= & 96 \\
\left|C_{H_{4}}(2 B)\right|= & 144
\end{aligned}\left(\begin{array}{ccccc}
1 & 1 & 1 & 1 \\
30 & f & g & h \\
30 & j & k & l \\
20 & n & o & p
\end{array}\right)
\end{aligned}
$$

In order to determine the entries $f, g, h, j, k, l, n, o$ and $p$ of the Fischer-Clifford matrix $M(2 A)$, the GAP output for programme D for the matrix $M(2 A)$ is first generated as,

$$
M\left(2 A^{\prime}\right)=\left(\begin{array}{rrrr}
1 & 1 & 1 & 1 \\
20 & 2 & 2 & -7 \\
30 & 3 & -6 & 3 \\
30 & -6 & 3 & 3
\end{array}\right)
$$


Clearly, row 2 of matrix $M\left(2 A^{\prime}\right)$ becomes row 4 of Fischer-Clifford matrix $M(2 A)$. Using the centralizer orders for the classes $6 A, 6 B$ and $6 C$ of $\bar{G}$, column 4 of $M\left(2 A^{\prime}\right)$ becomes column 2 of $M(2 A)$ and thus, $f=3, j=3$ and $n=7$. Now, columns 3 and 4 of matrix $M(2 A)$ are going to be identified using the fact that for any $p$-singular element $g$ of any finite group $G$ and irreducible character $\chi$ of $G$, then $\chi(g) \equiv \chi\left(g^{p}\right) \bmod p$. Noting from Table 3 that $\chi(6 B) \bmod 2 \equiv \chi(3 A)$, the second power map of the class $6 B$ of $\bar{G}$ is applied. If we suppose that column 3 in $M\left(2 A^{\prime}\right)$ becomes column 3 of $M(2 A)$, it turns out that $\chi(6 B) \bmod 2 \not \equiv \chi(3 A)$ for all irreducible characters of $\bar{G}$ on classes $3 A$ and $6 B$ of $\bar{G}$ and coming from inertia factor groups $H_{2}, H_{3}$ and $H_{4}$. Thus column 2 of matrix $M\left(2 A^{\prime}\right)$ becomes column 3 of $M(2 A)$ while column 3 of $M\left(2 A^{\prime}\right)$ automatically becomes column 4 of $M(2 A)$. The final Fischer-Clifford matrix assumes the following structure with $g=3, h=-6, k=-6, l=3, o=2$ and $p=2$.

$$
M(2 A)=\begin{aligned}
& 2880 \\
& 96 \\
& 96 \\
& 144
\end{aligned}\left(\begin{array}{cccc}
1 & 1 & 1 & 1 \\
30 & 3 & 3 & -6 \\
30 & 3 & -6 & 3 \\
20 & -7 & 2 & 2 \\
9 & 180 & 270 & 270
\end{array}\right)
$$

With a quite a number of the Fischer-Clifford matrices of $\bar{G}$ containing entries which involve the complex number $\alpha=-\frac{1}{2}+\frac{\sqrt{-3}}{2}$ such that $\alpha^{3}=$ 1 , the Fischer-Clifford matrix corresponding to the conjugacy class $6 D$ of $G=L_{4}(3)$ is briefly discussed. Using theorem 5.2.4 and property (e) in [18], $M(6 D)$ has the following form with corresponding weights attached to the rows and columns:

\begin{tabular}{|c|c|c|c|c|c|c|c|}
\hline & & $\left|C_{\bar{G}}(6 T)\right|$ & $\left|C_{\bar{G}}(6 U)\right|$ & $\left|C_{\bar{G}}(6 V)\right|$ & $\left|C_{\bar{G}}(6 W)\right|$ & $\left|C_{\bar{G}}(6 X)\right|$ & $\left|C_{\bar{G}}(6 Y)\right|$ \\
\hline & & 324 & 324 & 324 & 162 & 162 & 162 \\
\hline$\left|C_{H_{1}}(6 D)\right|=$ & 36 & 1 & 1 & 1 & 1 & 1 & 1 \\
\hline$\left|C_{H_{2}}(6 D)\right|=$ & 36 & 1 & $a$ & $f$ & $k$ & $p$ & $u$ \\
\hline$\left|C_{H_{3}}(6 E)\right|=$ & 36 & 1 & $b$ & $g$ & $l$ & $q$ & $v$ \\
\hline$\left|C_{H_{4}}(6 A)\right|=$ & 18 & 2 & $c$ & $h$ & $m$ & $r$ & $w$ \\
\hline$\left|C_{H_{4}}(6 F)\right|=$ & 18 & 2 & $d$ & $i$ & $n$ & $s$ & $x$ \\
\hline$\left|C_{H_{4}}(6 J)\right|=$ & 18 & 2 & $e$ & $j$ & $o$ & $t$ & $y$ \\
\hline & $m_{j}$ & 81 & 81 & 81 & 162 & 162 & 162 \\
\hline
\end{tabular}


To determine the remaining entries of the Fischer-Clifford matrix $M(6 D)$, the GAP output for programme $\mathrm{D}$ for $M(6 D)$ is computed,

$$
M\left(6 D^{\prime}\right)=\left(\begin{array}{cccccc}
1 & 1 & 1 & 1 & 1 & 1 \\
2 & 2 & 2 & -1 & -1 & -1 \\
2 & 2 \bar{\alpha} & 2 \alpha & -\alpha & -1 & -\bar{\alpha} \\
1 & \bar{\alpha} & \alpha & \alpha & 1 & -\bar{\alpha} \\
2 & 2 \alpha & 2 \bar{\alpha} & -\bar{\alpha} & -1 & -\alpha \\
1 & \alpha & \bar{\alpha} & \bar{\alpha} & 1 & \alpha
\end{array}\right)
$$

where $\bar{\alpha}=-\frac{1}{2}-\frac{\sqrt{-3}}{2}$ and $\alpha=-\frac{1}{2}+\frac{\sqrt{-3}}{2}$. The rows of Fischer-Clifford matrix $M\left(6 D^{\prime}\right)$ are then rearranged to match the structure above. Thus we have

$$
M\left(6 D^{\prime}\right)=\left(\begin{array}{cccccc}
1 & 1 & 1 & 1 & 1 & 1 \\
1 & \bar{\alpha} & \alpha & \alpha & 1 & -\bar{\alpha} \\
1 & \alpha & \bar{\alpha} & \bar{\alpha} & 1 & \alpha \\
2 & 2 & 2 & -1 & -1 & -1 \\
2 & 2 \bar{\alpha} & 2 \alpha & -\alpha & -1 & -\bar{\alpha} \\
2 & 2 \alpha & 2 \bar{\alpha} & -\bar{\alpha} & -1 & -\alpha
\end{array}\right)
$$

Taking into consideration properties of Fischer-Clifford matrices discussed in [6], [13], [14] and [23], the values of the irreducible characters of $\bar{G}$ on the classes $3 R, 3 P, 3 Q$ and $3 S$ that have already been obtained by Fischer-Clifford matrix $M(3 C)$, and using the fact that $(6 U)^{2}=3 R$, $(6 V)^{2}=3 R,(6 W)^{2}=3 P,(6 X)^{2}=3 Q$ and $(6 Y)^{2}=3 S$ (see Table 3), the final Fischer-Clifford matrix $M(6 D)$ assumes the form below.

$\begin{array}{llllll}324 & 324 & 324 & 162 & 162 & 162\end{array}$

$$
M(6 D)=\begin{aligned}
& 36 \\
& 36 \\
& 36 \\
& 18 \\
& 18 \\
& 18
\end{aligned}\left(\begin{array}{cccccc}
1 & 1 & 1 & 1 & 1 & 1 \\
1 & \alpha & \bar{\alpha} & \alpha & \bar{\alpha} & 1 \\
1 & \bar{\alpha} & \alpha & \bar{\alpha} & \alpha & 1 \\
2 & 2 & 2 & -1 & -1 & -1 \\
2 & 2 \bar{\alpha} & 2 \alpha & -\bar{\alpha} & -\alpha & -1 \\
2 & 2 \alpha & 2 \bar{\alpha} & -\alpha & -\bar{\alpha} & -1
\end{array}\right)
$$

For each class representative $g \in L_{4}(3)$, a Fischer-Clifford matrix $M(g)$ is constructed and listed in Table 7. 
TABLE 7. The Fischer-Clifford Matrices of $\bar{G}=3^{6}: L_{4}(3)$

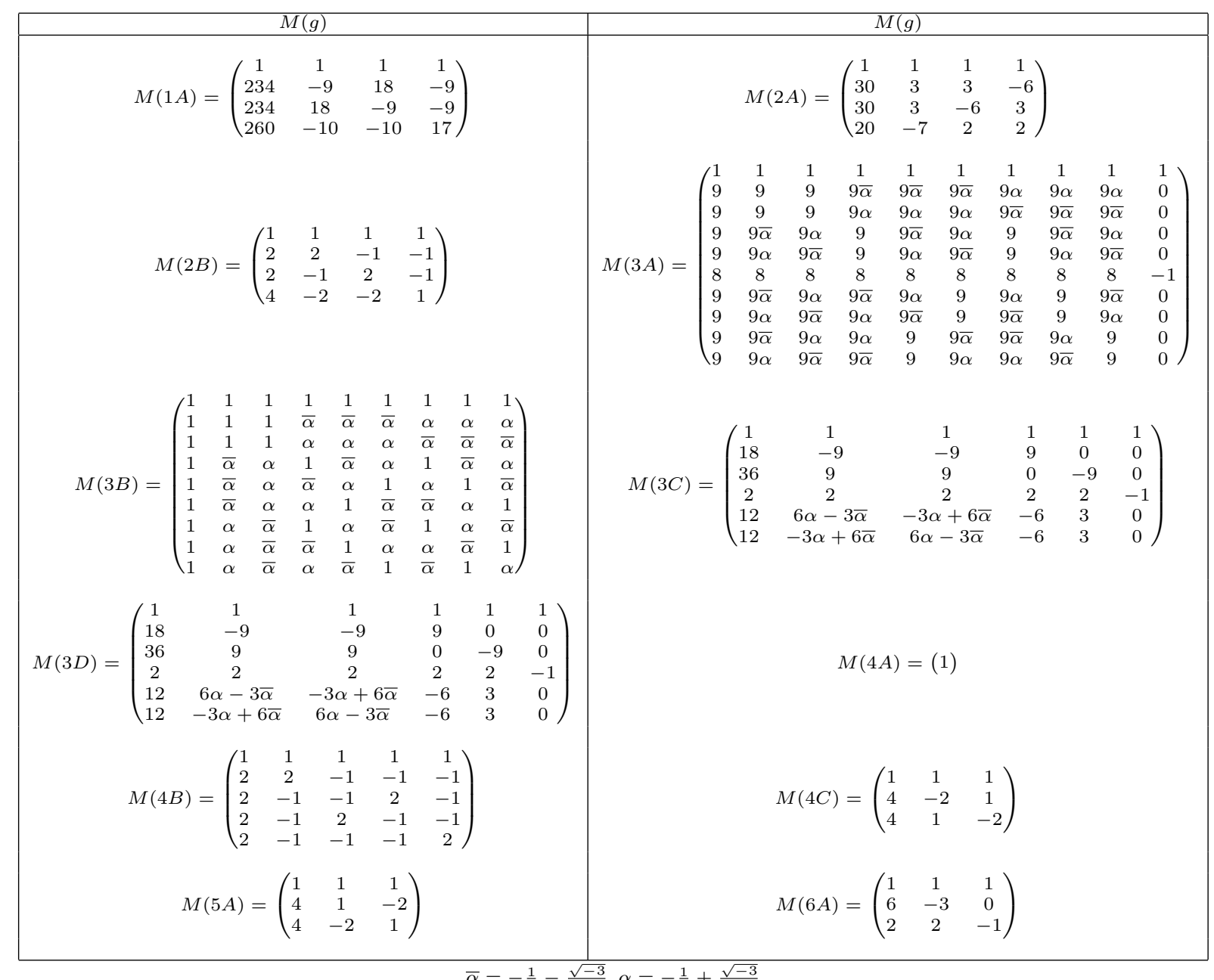


TABLE 7. (continued)

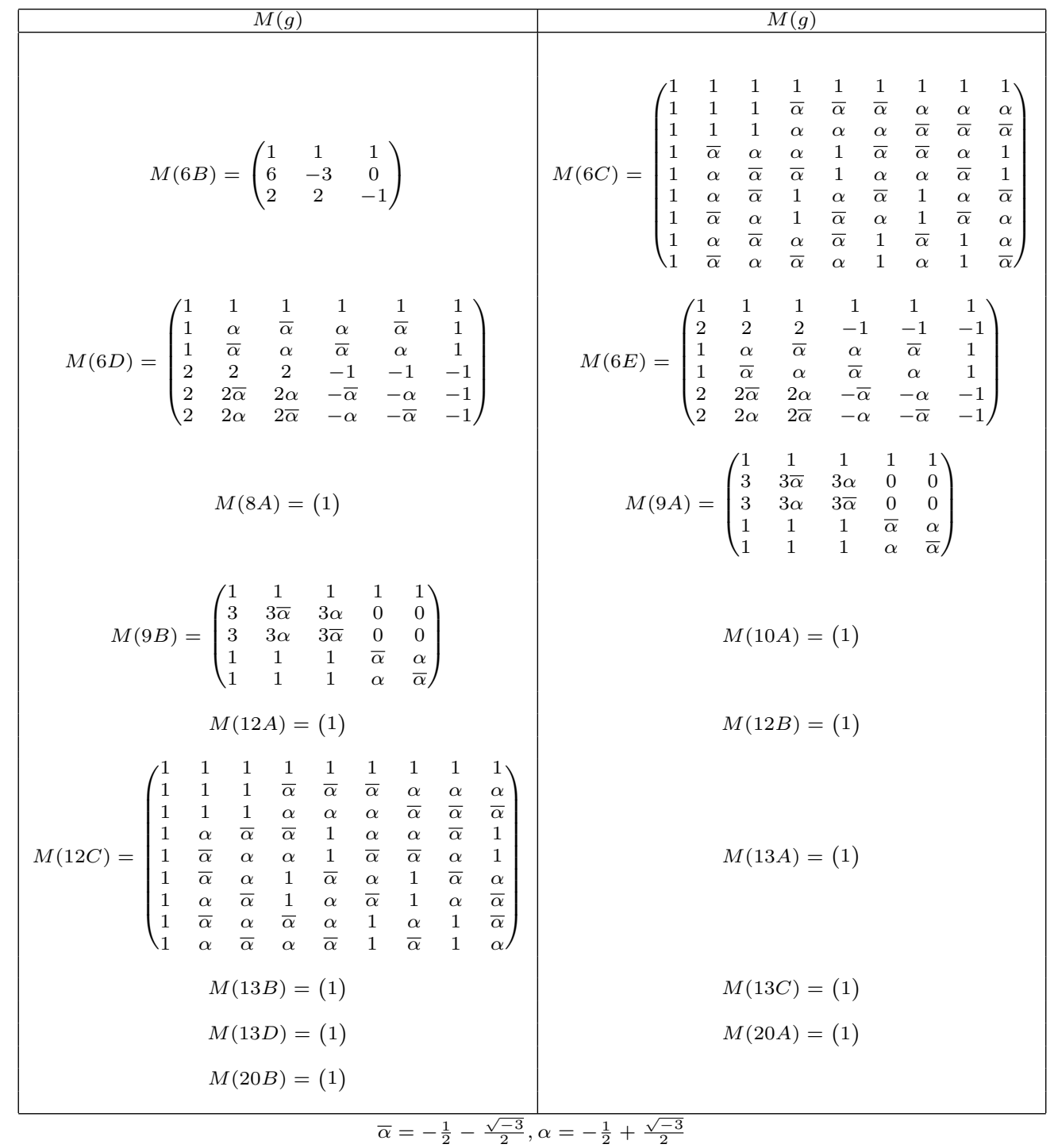




\section{The Character Table of $\bar{G}=3^{6}: L_{4}(3)$}

Having obtained the conjugacy classes of $\bar{G}=3^{6}: L_{4}(3)$, the ordinary character tables of all the inertia factor groups available in GAP [11], the fusions of conjugacy classes of the inertia factors into classes of $L_{4}(3)$ and the Fischer-Clifford matrices of $\bar{G}=3^{6}: L_{4}(3)$, the full character table of $\bar{G}$ can now be constructed by following the theoretical outline discussed in Section 2. This character table is a $111 \times 111$ complex-valued matrix partitioned row-wise into four blocks $\triangle_{1}, \triangle_{2}, \triangle_{3}$ and $\triangle_{4}$, where each block corresponds to an inertia group $\bar{H}_{i}=3^{6}: H_{i}$. In fact, $\triangle_{1}=\left\{\chi_{i} \mid 1 \leq i \leq 29\right\}$, $\triangle_{2}=\left\{\chi_{i} \mid 30 \leq i \leq 49\right\}, \triangle_{3}=\left\{\chi_{i} \mid 50 \leq i \leq 69\right\}$ and $\triangle_{4}=\left\{\chi_{i} \mid 70 \leq i \leq\right.$ $111\}$, where $\operatorname{Irr}\left(3^{6}: L_{4}(3)\right)=\cup_{i=1}^{4} \triangle_{i}$. The consistency and accuracy of this table has been tested using Programme $\mathrm{C}$ in [22] and the complete character table of the group $\bar{G}$ can be accessed via the link below.

https://drive.google.com/file/d/16oDwruWA0qWOFJNy8JmgOoESw7UoumVR/view?usp=sharing

\section{The Fusion of $3^{6}: L_{4}(3)$ of into $O_{8}^{+}(3)$}

Since $\bar{G}=3^{6}: L_{4}(3)$ is a maximal subgroup of $O_{8}^{+}(3)$ of index 1120 , then the action of $O_{8}^{+}(3)$ on the cosets of $\bar{G}$ gives rise to a permutation character $\chi\left(O_{8}^{+}(3) \mid \bar{G}\right)$ of degree 1120 . From the ATLAS of finite groups [8], $\chi\left(O_{8}^{+}(3) \mid \bar{G}\right)=1 a+300 a+819 a$, where $1 a, 300 a$ and $819 a$ are irreducible characters of $O_{8}^{+}(3)$ of degrees 1,300 and 819 respectively. Using the information provided by the conjugacy classes of $3^{6}: L_{4}(3)$ and $O_{8}^{+}(3)$, the power maps and the permutation character of $O_{8}^{+}(3)$ of degree 1120 , a partial fusion of $\bar{G}$ into $O_{8}^{+}(3)$ is obtained. To complete the fusion maps, irreducible characters of $O_{8}^{+}(3)$ of small degrees are restricted to $3^{6}: L_{4}(3)$. To determine the restrictions of irreducible characters of $O_{8}^{+}(3)$ to $3^{6}: L_{4}(3)$, the technique of set intersections for characters which has been discussed in detail in [1], [17] and [18] is used.

Let $\rho$ be the character of $L_{4}(3)$ afforded by the regular representation of $L_{4}(3)$. Then $\rho=\sum_{i=1}^{29} \varepsilon_{i} \phi_{i}$, where $\phi_{i} \in \operatorname{Irr}\left(L_{4}(3)\right)$ and $\varepsilon_{i}=\operatorname{deg}\left(\phi_{i}\right)$. Thus $\rho$ can be regarded as a character of $3^{6}: L_{4}(3)$ which contains $3^{6}$ in its kernel such that,

$$
\rho(g)= \begin{cases}\left|L_{4}(3)\right| & \text { if } g \in 3^{6} \\ 0 & \text { otherwise }\end{cases}
$$


If $\psi$ is a character of $O_{8}^{+}(3)$, then

$$
\begin{aligned}
\langle\rho, \psi\rangle_{\bar{G}} & =\frac{1}{\mid 3^{6}: L_{4}(3)}\{\rho(1 A) \psi(1 A)+234 \rho(3 A) \psi(3 A)+234 \rho(3 B) \psi(3 B)+260 \rho(3 C) \psi(3 C)\} \\
& =\frac{1}{3^{6}: L_{4}(3)}\left\{\left|L_{4}(3)\right| \psi(1 A)+234\left|L_{4}(3)\right| \psi(3 A)+234\left|L_{4}(3)\right| \psi(3 B)+260\left|L_{4}(3)\right| \psi(3 C)\right\} \\
& =\frac{1}{729}\{\psi(1 A)+234 \psi(3 A)+234 \psi(3 B)+260 \psi(3 C)\} \\
& =\left\langle\psi_{3^{6}}, \tau_{1}\right\rangle
\end{aligned}
$$

where $\tau_{1}$ is the identity character of $3^{6}$ and $\psi_{3^{6}}$ is the restriction of $\psi$ to $3^{6}$. Also for $\psi$ it is obtained that;

$$
\psi_{3^{6}}=a_{1} \theta_{1}+a_{2} \theta_{2}+a_{3} \theta_{3}+a_{4} \theta_{4}
$$

where $a_{1}, a_{2}, a_{3}, a_{4} \in \mathbf{N} \cup\{0\}$ and $\theta_{i}, i \in\{1,2,3,4\}$ are sums of the irreducible characters of $3^{6}$ which are in one orbit under action of $L_{4}(3)$ on $\operatorname{Irr}\left(3^{6}\right)$. Letting $\tau_{j} \in \operatorname{Irr}\left(3^{6}\right)$, where $j \in\{1,2, \ldots, 729\}$, then,

$$
\begin{array}{cc}
\theta_{1} & =\tau_{1}, \operatorname{deg}\left(\theta_{1}\right)=1 \\
\theta_{2} & =\sum_{j=2}^{235} \tau_{j}, \operatorname{deg}\left(\theta_{2}\right)=234 \\
\theta_{3} & =\sum_{j=236}^{469} \tau_{j}, \operatorname{deg}\left(\theta_{3}\right)=234 \\
\theta_{4}=\sum_{j=470}^{729} \tau_{j}, \operatorname{deg}\left(\theta_{4}\right)=260
\end{array}
$$

Hence

$$
\psi_{3^{6}}=a_{1} \tau_{1}+a_{2} \sum_{j=2}^{235} \tau_{j}+a_{3} \sum_{j=236}^{469} \tau_{j}+a_{4} \sum_{j=470}^{729} \tau_{j}
$$

and,

$$
\begin{gathered}
\left\langle\psi_{3^{6}}, \psi_{3^{6}}\right\rangle=a_{1}^{2}+234 a_{2}^{2}+234 a_{3}^{2}+260 a_{4}^{2} \\
=\frac{1}{729}\{\psi(1 A) \psi(1 A)+234 \psi(3 A) \psi(3 A)+234 \psi(3 B) \psi(3 B)+260 \psi(3 C) \psi(3 C)\},
\end{gathered}
$$

where

$$
a_{1}=\left\langle\psi_{3^{6}}, \tau_{1}\right\rangle=\langle\rho, \psi\rangle_{\bar{G}}
$$

Now, the above results are applied to some irreducible characters of $O_{8}^{+}(3)$ of small degrees, which in this case are $\psi_{1}=260 a, \psi_{2}=260 b$, $\psi_{3}=260 c, \psi_{4}=819 b, \psi_{5}=819 c, \psi_{6}=2275 a, \psi_{7}=2275 b, \psi_{8}=2275 c$, and $\psi_{9}=2275 d$ of degrees 1, 260, 260, 260, 819, 819, 2275, 2275, 2275, and 2275 , respectively (see ATLAS for character table of $O_{8}^{+}(3)$ ). From the partial fusion that has already been determined, the class $3 C$ of $\bar{G}$ must fuse into the class $3 A$ of $O_{8}^{+}(3)$. Also, the classes $3 A$ and $3 B$ of $\bar{G}$ must each fuse into one of the following classes, $3 B, 3 C, 3 D, 3 E, 3 F$ and $3 G$ of $O_{8}^{+}(3)$ such that the condition $a_{1}=\left\langle\psi_{3^{6}}, \tau_{1}\right\rangle \in \mathbf{N} \cup\{0\}$ is satisfied. The values of $\psi_{1}=260 a$ on the classes $3 A, 3 B$ and $3 C$ of $O_{8}^{+}(3)$ violate this condition. The only combination that satisfies this condition is $3 A, 3 B$ and $3 E$ and thus for $\psi_{1}$ the following result is obtained, 


$$
a_{1}=\langle\rho, \psi\rangle_{\bar{G}}=\frac{1}{729}\{260+234(17)+234(44)+260(17)\}=26
$$

Since the degree of $\psi_{1}$ is 260 then,

$$
a_{1}+234 a_{2}+234 a_{3}+260 a_{4}=260
$$

and so we must have that $a_{1}=26, a_{2}=1, a_{3}=0$ and $a_{4}=0$ or $a_{1}=26$, $a_{2}=0, a_{3}=1$ and $a_{4}=0$. It turns out that $\left(\psi_{1}\right)_{3^{6}: L_{4}(3)}$ is a sum of two irreducible characters of $3^{6}: L_{4}(3)$ of degrees 26 and 234 , respectively. Based on the partial fusion of $3^{6}: L_{4}(3)$ into $O_{8}^{+}(3)$ that has already been done, the result below is obtained,

$$
\left(\psi_{1}\right)_{3^{6}: L_{4}(3)}=\chi_{3}+\chi_{50}
$$

Similarly, for $\psi_{2}=260 b, a_{1}$ is obtained as follows,

$$
a_{1}=\langle\rho, \psi\rangle_{\bar{G}}=\frac{1}{729}\{260+234(-10)+234(-10)+260(17)\}=0
$$

Since the degree of $\psi_{2}$ is 260 then

$$
a_{1}+234 a_{2}+234 a_{3}+260 a_{4}=260
$$

so that $a_{1}=0, a_{2}=0, a_{0}=0$ and $a_{4}=1$ and thus $\left(\psi_{2}\right)_{3^{6}: L_{4}(3)}$ is an irreducible character of $3^{6}: L_{4}(3)$ of degree 260 . Based on the partial fusion of $3^{6}: L_{4}(3)$ into $O_{8}^{+}(3)$ that has already been done, it turns out that

$$
\left(\psi_{2}\right)_{3^{6}: L_{4}(3)}=\chi_{71} \text {. }
$$

Applying the same procedure to $\psi_{3}, \psi_{4}, \psi_{5}, \psi_{6}, \psi_{7}, \psi_{8}$ and $\psi_{9}$, we obtained the restricted characters below.

$$
\begin{array}{ll}
\left(\psi_{3}\right)_{3^{6}: L_{4}(3)} & =\chi_{76} \\
\left(\psi_{4}\right)_{3^{6}: L_{4}(3)} & =\chi_{4}+\chi_{79} \\
\left(\psi_{5}\right)_{3^{6}: L_{4}(3)} & =\chi_{4}+\chi_{80} \\
\left(\psi_{6}\right)_{3^{6}: L_{4}(3)} & =\chi_{6}+\chi_{32}+\chi_{90} \\
\left(\psi_{7}\right)_{3^{6}: L_{4}(3)} & =\chi_{6}+\chi_{31}+\chi_{91} \\
\left(\psi_{8}\right)_{3^{6}: L_{4}(3)} & =\chi_{7}+\chi_{51}+\chi_{87} \\
\left(\psi_{9}\right)_{3^{6}: L_{4}(3)} & =\chi_{7}+\chi_{52}+\chi_{86}
\end{array}
$$

By making use of the partial fusion which has already been determined, the values of $\psi_{1}, \ldots, \psi_{9}$ on the classes of $O_{8}^{+}(3)$ and the values of $\left(\psi_{1}\right)_{3^{6}: L_{4}(3)}, \ldots,\left(\psi_{9}\right)_{3^{6}: L_{4}(3)}$ on the classes of $3^{6}: L_{4}(3)$, the fusion of $3^{6}: L_{4}(3)$ into $O_{8}^{+}(3)$ is completed and is found in Table 3 . 


\section{Acknowledgments}

The first author really want to thanks God for all His blessings especially in guiding his research towards the writing of this paper and also want to show appreciation to all of his supervisors for their invaluable and timely input. May God bless you all abundantly. This research was supported by the Faculty of Science at Nelson Mandela University [Cost centre: GB85 and account number: 4920].

\section{References}

[1] F. Ali, Fischer-Clifford theory for split and non-split group extensions, PhD. Thesis, University of Natal, 2001.

[2] A. B. M. Basheer, and J. Moori, "A survey on Clifford-Fischer theory," in Groups St Andrews 2013, C. M. Campbell, M. R. Quick, E. F. Robertson, and C. M Roney-Dougal, Eds. Cambridge: Cambridge University Press, 2015, pp. 160-172.

[3] A. B. Basheer and T. T. Seretlo, "On a group of the form $2^{14}: \operatorname{Sp}(6,2)$ ", Quaestiones mathematicae, vol. 39, no. 1, pp. 45-57, 2015. doi: 10.2989/16073606.2015.1023865

[4] A. Basheer and J. Moori, "On a group of the form $3^{7}: \operatorname{Sp}(6,2)$ ", International journal of group theory, vol. 5, no. 2, pp. 41-59, 2016. doi: 10.22108/ IJGT.2016.8047

[5] C. Chileshe, Irreducible characters of Sylow p-Subgroups associated with some classical linear groups, PhD. Thesis, North-West University, 2016.

[6] O. Bonten, Clifford-Matrizen, Diplomarbeit, Lehrstuhl D für Mathematik, RWTH Aachen, 1988.

[7] W. Bosma and J. J. Canon (Eds.), Handbook of magma functions, Sidney: University of Sydney, 1994.

[8] J. H. Conway, R. T. Curtis, S. P. Norton, R. A. Parker and R. A. Wilson, Atlas of finite groups, Oxford: Oxford University Press, 1985.

[9] B. Fischer, "Clifford-matrices", in Representation Theory of Finite Groups and Finite-Dimensional Algebras, G. O. Michler and C. M. Ringel. Eds. Birkhäuser: Springer, 1991, pp. 1-16. 
[10] P. X. Gallagher, "Group characters and normal hall subgroups”, Nagoya mathematical journal, vol. 21, pp. 223-230, 1962. doi: 10.1017/ s0027763000023849

[11] "GAP system for computational discrete algebra. version 4.11.0," GAP System for Computational Discrete Algebra, Feb-2020. [Online]. Available: https:/ / www.gap-system.org/ Releases/ 4.11.0.html.

[12] I. M. Isaacs, Character theory of finite groups, San Diego: Academic Press, 1976.

[13] R. J. List, "On the characters of $2^{n-\epsilon} \cdot S_{n}$ ", Archiv der mathematik, vol. 51, no. 2, pp. 118-124, 1988. doi: 10.1007/ bf01206468

[14] R. List and I. Mahmoud, "Fischer matrices for wreath products $\mathrm{GwS}_{\mathrm{n}}$ ", Archiv der mathematik, vol. 50, no. 5, pp. 394-401, 1988. doi: 10.1007/ bf01196499

[15] J. Moori and T. Seretlo, "On 2 nonsplit extension groups associated with HS and HS:2", Turkish journal of mathematics, vol. 38, pp. 60-78, 2014. doi: 10.3906/ mat-1209-11

[16] J. Moori and T. Seretlo, "On the Fischer-Clifford matrices of a maximal subgroup of the Lyons group Ly", Bulletin of the Iranian mathematical Society, vol. 39, no. 5, pp. 1037-1052, 2013. [On line]. Available: https:/ / bit.ly/ 3GIAqc6

[17] J. Moori, On the Groups $\mathrm{G}^{+}$and $\overline{\mathrm{G}}$ of the forms $2^{10}: \mathrm{M}_{22}$ and $2^{10}: \mathrm{M}_{22}, \mathrm{PhD}$. thesis, University of Birmingham, 1975.

[18] Z. E. Mpono, Fischer-Clifford Theory and Character Tables of Group Extensions, PhD. thesis, University of Natal, 1998.

[19] A.L. Prins, "A maximal subgroup $2^{4+6}:\left(A_{5} \times 3\right)$ of $G_{2}(4)$ treated as a non-split extension $\overline{\mathrm{G}}=2^{6 \cdot}\left(2^{4}:\left(A_{5} \times 3\right)\right)$ ", Advances in Group Theory and Applications, vol. 10, pp. 43-66, 2020. doi: 10.32037/ agta-2020-009

[20] A. L. Prins, R. L. Monaledi and R. L. Fray, "On a maximal sub- group $\left(2^{9}: \mathrm{L}_{3}(4)\right): 3$ of the automorphism group $\mathrm{U}_{6}(2): 3$ of $\mathrm{U}_{6}(2) "$ ", Afrika matematika, vol. 31, no. 7-8, pp. 1311-1336, 2020. doi: 10.1007/ s13370-020-00798-x

[21] A. L. Prins, "The character table of an involution centralizer in the Dempwolff group $2^{5 \cdot} \mathrm{GL}_{5}(2) "$ ", Quaestiones mathematicae, vol. 39, no. 4, pp. 561-576, 2015. doi: 10.2989/ 16073606.2015.1113210

[22] A. L. Prins, Fischer-Clifford matrices and character tables of inertia groups of maximal subgroups of finite simple groups of extension type, PhD. thesis, University of the Western Cape, 2011. 
[23] R. B. Salleh, On the construction of the character tables of extension groups, PhD. thesis, University of Birmingham, 1982.

[24] T. T. Seretlo, Fischer Clif6rd Matrices and Character Tables of Cer- tain G roups A ssociated with Simple G roups $O_{10}^{+}(2)$, HS and Ly, PhD. thesis, University of KwaZulu Natal, 2011.

[25] R. A. Wilson, P. Walsh, J. Tripp, I. Suleiman, S. Rogers, R. Parker, S. Norton, S. Nickerson, S. Linton, J. Bray and R. Abbot, ATLAS of finite group representations. [On line]. Available: https:/ / brauer.maths.qmul. ac.uk/ Atlas/ v3/ 


\title{
David Mwanzia Musyoka
}

Department of Mathematics and Actuarial Science,

Kenyatta University,

P. O. Box 43844-00100,

Nairobi,

Kenya

e-mail: davidmusyoka21@yahoo.com

\section{Lydia Njuguna}

Department of Mathematics and Actuarial Science,

Kenyatta University,

P. O. Box 43844-00100,

Nairobi,

Kenya

e-mail: njuguna.lydia@ku.ac.ke

lydiahnjuguna@yahoo.com

\author{
Abraham Love Prins \\ Department of Mathematics and Applied Mathematics, \\ Faculty of Science, \\ Nelson Mandela University, \\ P. O. Box 77000, \\ Port Elizabeth, 6031, \\ South Africa \\ e-mail: abraham.prins@mandela.ac.za \\ abrahamprinsie@yahoo.com \\ Corresponding author \\ and

\section{Lucy Chikamai} \\ Department of Mathematics and Actuarial Science, \\ Kibabii University, \\ P. O. Box 1699-50200, \\ Bungoma, \\ Kenya \\ e-mail: chikamail@kibu.ac.ke \\ lucychikamai@gmail.com
}

